\title{
Epidemiology of trigeminal neuralgia: an electronic population health data study in Korea
}

\author{
Cheol-Hyeong Lee ${ }^{1}$, Ho-Yeon Jang ${ }^{1}$, Hyung-Sun Won ${ }^{2,3}$, Ja-Sook Kim ${ }^{4}$, and Yeon-Dong Kim ${ }^{1,3}$ \\ 'Department of Anesthesiology and Pain Medicine, Wonkwang University Hospital, Wonkwang University School of Medicine, Iksan, Korea \\ 2Department of Anatomy, Wonkwang University School of Medicine, Iksan, Korea \\ 3Jesaeng-Euise Clinical Anatomy Center, Wonkwang University School of Medicine, Iksan, Korea \\ ${ }^{4}$ Department of Nursing, Kunsan National University, Gunsan, Korea
}

Received February 9, 2021

Revised April 14, 2021

Accepted May 4, 2021

Handling Editor: Jeong-Gill Leem

\section{Correspondence}

Yeon-Dong Kim

Department of Anesthesiology and Pain Medicine, Wonkwang University School of Medicine, 460 Iksan-daero, Iksan 54538 , Korea

Tel: +82-63-859-1562

Fax: +82-63-859-5472

E-mail: kydpain@hanmail.net

Ja-Sook Kim

Department of Nursing, Kunsan National University, 558 Daehak-ro, Gunsan 54150, Korea

Tel: +82-63-469-1997

Fax: +82-63-469-1997

E-mail: jskim-98@hanmail.net
Background: Trigeminal neuralgia (TN) is one of the most painful disorder in the orofacial region, and many patients have suffered from this disease. For the effective management of TN, fundamental epidemiologic data related to the target population group are essential. Thus, this study was performed to clarify the epidemiological characteristics of TN in the Korean population. This is the first national study to investigate the prevalence of TN in Korean patients.

Methods: From 2014 to 2018, population-based medical data for 51,276,314 subscribers to the National Health Insurance Service of Korea were used for this study. Results: The incidence of TN was 100.21 per 100,000 person-years in the year of 2018 in Korea, and the male to female ratio was 1:2.14. The age group of 51-59 years had the highest prevalence of TN. Constant increases in medical cost, regional imbalance, and differences in prescription patterns by the medical specialties were showed in the management of TN.

Conclusions: The results in this study will not only help to study the characteristics of TN, but also serve as an important basis for the effective management of TN in Korea.

Key Words: Big Data; Cohort Studies; Epidemiologic Studies; Facial Pain; Incidence; National Health Programs; Patient Acceptance of Health Care; Population Surveillance; Prevalence; Republic of Korea; Trigeminal Neuralgia.

\section{INTRODUCTION}

Trigeminal neuralgia (TN) is a disease entity defined as "recurrent unilateral brief electric shock-like pains, abrupt in onset and termination, limited to the distribution of one or more divisions of the trigeminal nerve and triggered by innocuous stimuli." [1]. TN has been reported as one of the most painful disorder in the orofacial region, which has pain lasting from a second to few minutes [2]. Vascular compression of the trigeminal nerve has been known to be a major cause of TN, but TN can be also caused by either idiopathic conditions or lesions including cysts, tumors, and multiple sclerosis [3]. The diagnosis of TN is commonly established by clinically characterized feature, such as sudden severe facial pain rather than objective imaging studies, except only for the TN cases related to vascular compression [4]. Many cases of TN have thus frequently been misdiagnosed as other types of orofacial pain, which leads to unnecessary treatments for the patients [5].

For managing $\mathrm{TN}$, most patients are commonly pre- (c) This is an open-access article distributed under the terms of the Creative Commons Attribution Non-Commercial License (http://creativecommons.org/licenses/by-nc/4.0/), which permits unrestricted non-commercial use, distribution, and reproduction in any medium, provided the original work is properly cited.

(C) The Korean Pain Society, 2021
Author contributions: Cheol-Hyeong Lee: Writing/manuscript preparation; Ho-Yeon Jang: Data curation; Hyung-Sun Won: Writing/manuscript preparation; Ja-Sook Kim: Writing/manuscript preparation; Yeon-Dong Kim: Writing/manuscript preparation. 
scribed medications, and surgical interventions are also required in some cases [6]. However, paroxysmal attack and intractable pain are still challenging conditions for both physicians and patients. TN often presents with symptoms such as depression, anxiety, or sleep disorders resulting from chronic severe pain [7]. The daily activities of the patient are also affected, which degrades the quality of not only the patient's life, but also that of their families. The frequent use of health care resources for the management of this disease also increases its socio-economic burden [8].

In clinical practice, the management of $\mathrm{TN}$ surpassed US $\$ 94$ million in the United States between 2003 and 2013 [9]. TN is a rare disease and scant high-quality epidemiological data exist regarding this disease. There has yet to be consensus in the literature regarding the prevalence of $\mathrm{TN}$, with only a few studies on a limited study group $[10,11]$. Thus, fundamental epidemiologic data are essential to establish the effective management of TN. The aim of this study was to identify the epidemiological features of $\mathrm{TN}$, including incidence, regional distribution, and healthcare resource utilization based on the population data in Korea.

\section{MATERIALS AND METHODS}

This study was approved by the Institutional Review Board of Wonkwang University Hospital (IRB ID No. WKUH 2020-03-021) and was designed as a population-based cross-sectional study.

\section{Population}

Since 1989, all the residents of South Korea have been covered by the National Health Insurance Service (NHIS). The NHIS is a non-profit public insurance system under the Ministry of Health and Welfare that provides health insurance to all citizens living in Korea. The NHIS collects demographic data, including age, sex, and residential address, based on each patient's national identification number. Similarly, all medical data, such as disease codes, laboratory examinations, prescribed medications, and hospitalization details, are documented in a computerized database by the Health Insurance Review and Assessment Service (HIRA), which is a government organization established for medical billing purposes. Population-based medical data for $51,276,314$ subscribers to the NHIS and HIRA were analyzed in this study with a focus on the epidemiological features of TN in Korea. Data on items such as incidence and medical cost were analyzed between the years 2014 and 2018, and on others such as age, sex, regional distribution, healthcare resource utilization, and prescription patterns were analyzed using data from 2018.

\section{Data extraction}

The diagnostic code from HIRA was used for data collection from the electronic database. The HIRA code for TN (G500) was assigned based upon the World Health Organization's Ninth Revision, International Classification of Diseases. Prescription patterns of TN by medial specialty were analyzed using the Anatomical Therapeutic Chemical (ATC) code, which is used in the HIRA system, for the analysis of medication use. ATC is a drug classification system that classifies the active ingredients of drugs according to the organ or system on which they act and their therapeutic, chemical, and pharmacological properties [12]. In accordance with the ATC code classification, drugs prescribed for each patient related to TN were classified as follows: antiepileptic drugs, nonsteroidal anti-inflammatory drugs (NSAIDs), opioids, antidepressants, and muscle relaxants. The regional distribution was analyzed based on the national residence registration data from the Korean Statistical Information System of Statistics in this period.

Regarding healthcare resource utilization, the hospitals of the medical system in Korea are classified according to the medical facilities and personnel in this order; primary health care facilities (PHCFs), hospitals, general hospitals, and advanced general hospitals. PHCFs are usually outpatient-based clinics, including all medical specialties. An advanced general hospital is a hospital that provides tertiary care, capable of treating patients with more severe illnesses referred from PHCFs, hospitals, and general hospitals.

\section{RESULTS}

\section{Incidence of TN between 2014 and 2018}

In 2014, the number of patients with TN was 42,096 , and the number gradually increased to 51,403 in 2018. Based on collected data, the prevalence of TN by age group and sex was analyzed (Fig. 1). The prevalence was 82.11 per 100,000 person-years and it further increased to 100.26 in 2018 , with an increase rate of $22.1 \%$.

\section{Distribution of TN by age group and sex in 2018}

The prevalence of TN by age group and sex was analyzed (Fig. 2). The total number of patients in 2018 was 16,315 male and 35,058 female. This showed female predomi- 
nance, and the female to male ratio was 2.14:1. In both sexes, the highest prevalence was found in the age group of 51-59 years.

\section{Regional distribution of TN in 2018}

In each province of Korea, the number of cases was analyzed in consideration with the population density (Fig. 3). The incidence of $\mathrm{TN}$ in these provinces was highly variable in 2018. TN was more prevalent in the metropolitan areas of Seoul and Gyeonggi than in the other provinces.

\section{Medical specialty related to the management of TN in 2018}

Based on our data analysis regarding medical specialty, patients with TN were mostly managed by the department of neurology (51\%). The other departments that managed patients with TN in Korea in 2018 included, in order of frequency, the departments of otorhinolaryngology (14\%), neurosurgery (12\%), and anesthesiology and pain medi-

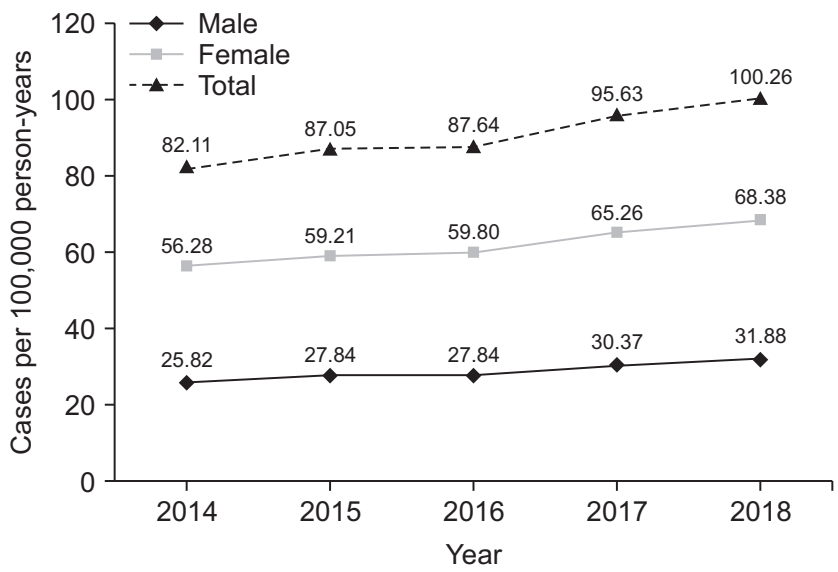

Fig. 1. Incidence of trigeminal neuralgia in Korea between 2014 and 2018. cine (6\%) (Fig. 4).

\section{Healthcare resource utilization and medical cost of TN in 2018}

More than $60 \%$ of patients with TN were managed in the PHCF. Advanced general hospitals accounted for $12 \%$ in the management of TN (Fig. 5). The medical costs per year between 2014 and 2018 showed an increasing trend from $3,910,766$ to $6,248,592$ USD (average exchange rate in 2014 to 2018: 1 USD = 1,137 Korean won) (Fig. 6). Medical cost per capita by each medical specialty was in the following order in 2018: neurosurgery, anesthesiology and pain medicine, neurology, internal medicine, and otorhinolaryngology. The average medical cost per patient per year for TN was USD 110. Patients with TN spent more than 326 USD in the department of neurosurgery, which was the highest cost among all medical specialties (Fig. 7).

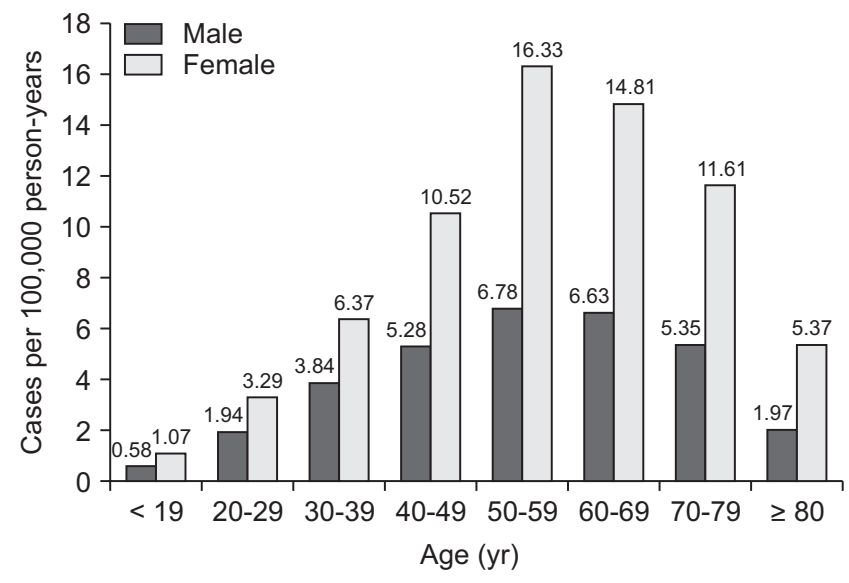

Fig. 2. Distribution of trigeminal neuralgia by age and sex groups in 2018.

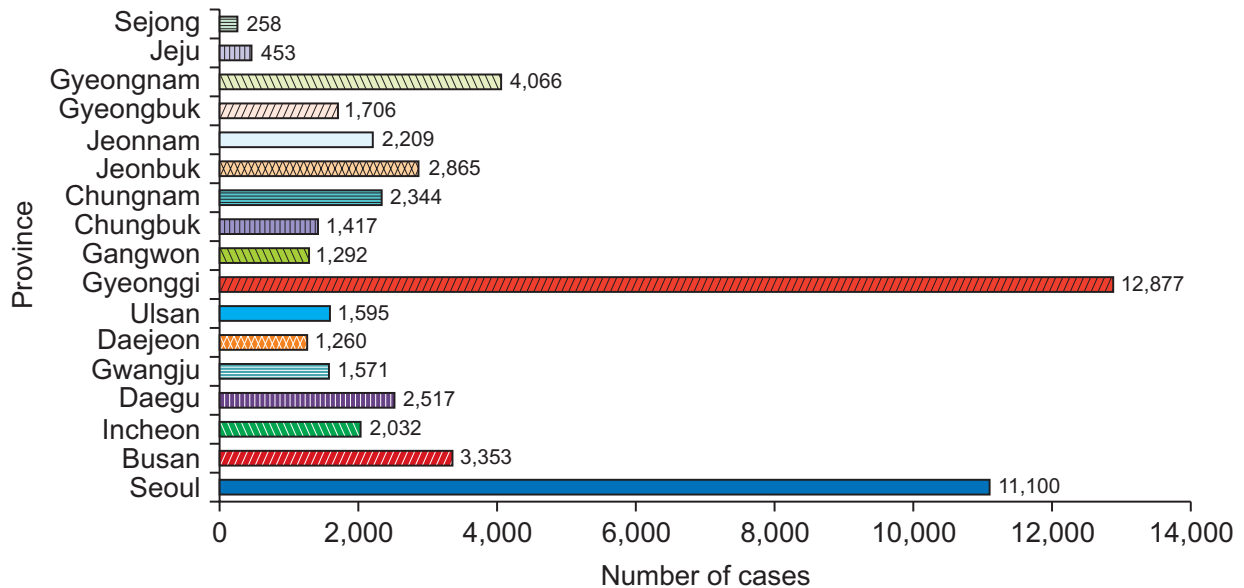

Korean J Pain 2021;34(3):332-338
Fig. 3. Regional distribution of trigeminal neuralgia in 2018. 


\section{Prescription patterns for TN in 2018}

Prescription patterns for the management of TN were analyzed (Fig. 8). Antiepileptic drugs were the most commonly used drug in the management of TN in Korea. There were some differences in the use of other drugs by medical specialties. Opioids and NSAIDs were most commonly prescribed in the department of anesthesia and pain medicine and department of otorhinolaryngology, respectively.

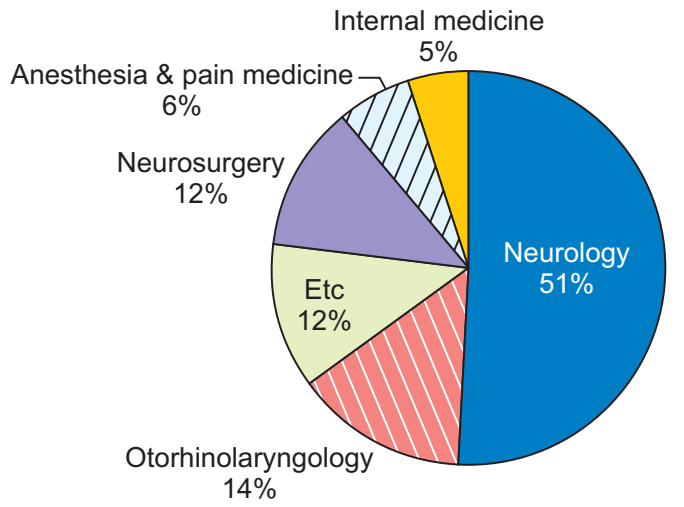

Fig. 4. Medical specialty related to the management of trigeminal neuralgia in 2018.

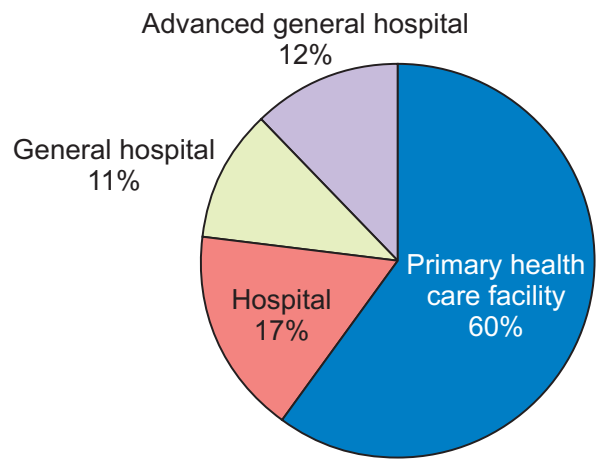

Fig. 5. Healthcare resource utilization for trigeminal neuralgia in 2018.

\section{DISCUSSION}

As TN is an unusual chronic pain due to its episodic, variable, and unpredictable nature, its prevalence is relatively

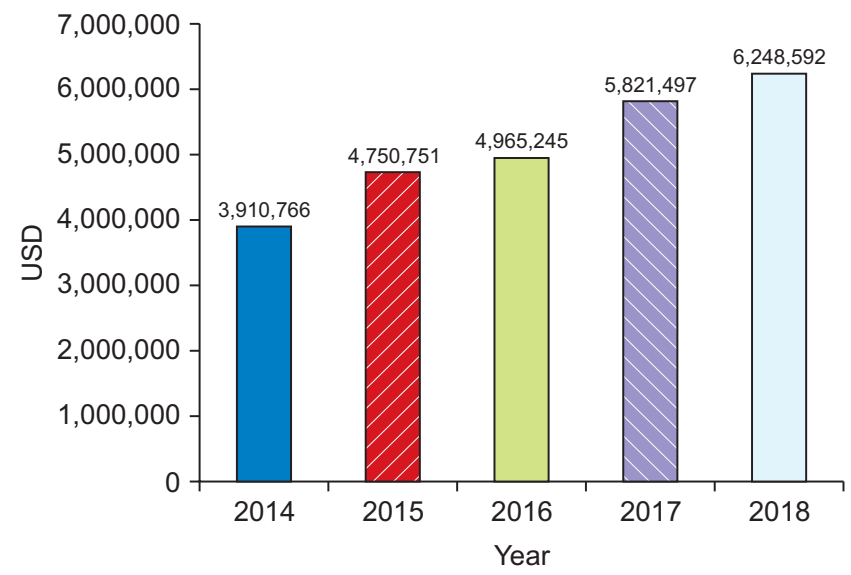

Fig. 6. Trend of medical cost for the management of trigeminal neuralgia between 2014 and 2018.

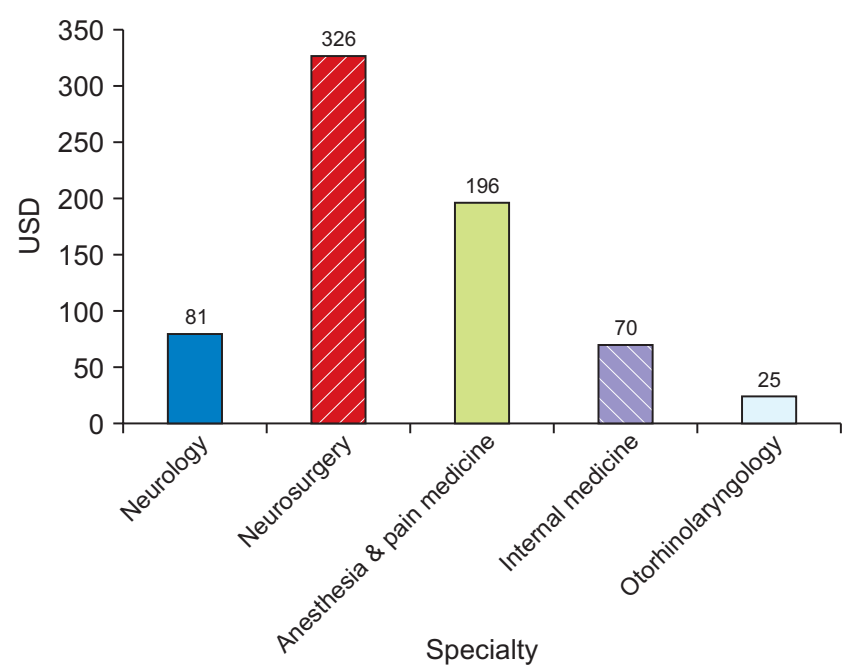

Fig. 7. Medical cost by the medical specialties in 2018.



Fig. 8. Prescription patterns for trigeminal neuralgia by medical specialty in 2018 . NSAIDs: nonsteroidal anti-inflammatory drugs. 
rarer than other neurogenic disorders. Up to now some retrospective studies have reported the epidemiology, incidence, and prevalence of $\mathrm{TN}$, but most of them focused on a restricted area [10] or Western countries [13-15]. A study in the US reported that the annual incidence of TN was 5.9 per 100,000 person-years in female and 3.4 per 100,000 person-years in male. The incidence increased with age, and female's incidence was slightly higher in all ages [14]. A retrospective cohort study in United Kingdom primary care reported the incidence of $\mathrm{TN}$ as 26.8 per 100,000 person-years [15]. A community-based study in Germany reported a lifetime prevalence of $0.3 \%$, and in Netherlands the incidence rate of TN was 21.7 per 100,000 person-years $[11,16]$. These studies were mostly based on limited populations centered on local hospitals. However, our study was the first national wide study using electronic population data with a larger study group, differentiating it from other studies. In the present study, the prevalence of TN was 100.25 per 100,000 person-years in 2018 , with the highest prevalence rate in both sexes in the age group of 51-59 years. The male to female ratio in the prevalence of TN was previously reported as being 1:1.9 [17] or 1:2.0 [18]. In the present study with larger study population, the ratio was 1:2.14. The differences in pain perception between the sexes have, as of yet, been poorly understood. In the previous study, the ways the brain responds to the pain were suggested as having sex differences in the pain stimulation [19]. In addition, there is a report on the existence of a significant association with gene polymorphism affecting serotonin release in the group of female suffering from $\mathrm{TN}$ [20].

In Korea, one study previously mentioned that the prevalence of TN was 81.8 per 100,000 person-years [21]. However, there was no consideration of the various epidemiological characteristics of $\mathrm{TN}$ in that study. In the present study, we used the most up-to-date data available data between 2014 and 2018 in Korea, with other various parameters such as regional distribution, medical cost, healthcare resource utilization, and prescription patterns by medical specialty. In view of the regional distribution, TN was more prevalent in the metropolitan areas, such as Seoul and Gyeonggi. Regarding the medical resource utilization, PHCFs were most commonly visited, followed by hospitals and general hospitals. Nobody knows the exact reason for the difference. This means that the primary physicians should have accurate knowledge of $\mathrm{TN}$ in the management of orofacial pain. Patients with TN were mostly managed by the department of neurology, followed by otorhinolaryngology, neurosurgery, anesthesia and pain medicine, and internal medicine. The medical cost for the treatment of TN in Korea has increased rapidly from 3,910,766 USD at the end of 2014 to 6,248,592 USD in 2018, with an increase of $159.77 \%$. Interventional treatment is recommended in clinical practice for the management of cases that are refractory to conventional pharmacological treatment [22]. The medical costs were highest in the department of neurosurgery and anesthesia and pain medicine, where interventional treatments (nerve blocks or surgery) are the major modes of management, which differs from the other departments. For effective management of TN, guidelines on primary care and referral to specialists will be helpful. In addition, various recognized clinically effective interventional treatments should be known as other treatment options.

Management of TN should initially begin with a pharmacologic approach, in the form of monotherapy. In refractory cases, combination therapy, using different kinds of drugs, could be also used. Anticonvulsants, including carbamazepine and oxycarbazepine, have been known to be first line medications [23]. To the best of our knowledge, to date, there has been no study on the prescription patterns for the management of TN. Based on our results, the most commonly prescribed drug in Korea was an anticonvulsant drug, followed by NSAIDs and opioids. Anticonvulsants, such as carbamazepine and oxcarbazepine, are the drugs of choice for the pharmacologic management of TN [24]. They inhibit the function of voltage-sensitive sodium channels in hyperexcited neuronal membranes, thus raising the threshold of excitability inhibiting the high frequency repetitive neuronal firing characteristic of TN [25]. In contrast, there is no evidence to support the use of NSAIDs for the treatment of neuropathic pain [26]. Moreover, NSAIDs are not recommended for longterm administration to treat patients with neuropathic pain syndrome because of side effects such as adverse cardiovascular events [27]. Nevertheless, our study found that NSAIDs were the second most prescribed drugs for TN. More research on the risks and benefits of NSAIDs in drug treatment of $\mathrm{TN}$ may be needed. Opioids can reduce pain during short-term use for the management of chronic neuropathic pain; however, the review of clinical evidence found insufficient evidence to determine whether pain relief continues and long-term opioid therapy improves function or quality of life [28]. Further, this study showed that the use of opioids as an adjunct to antiepileptic drugs is prevalent in patients with $\mathrm{TN}$, despite the lack of evidence regarding the efficacy of neuropathic pain management and the risk of abuse, addiction, and diversion [29].

This study had some limitations. First, this study does not include personal data because they are based on HIRA. In other words, individual patients are not traceable, and the disease characteristics of individuals are unknown. The prevalence could have been undervalued or overstated because the number of patients was counted only by 
the diagnostic code. Second, although many patients with TN may initially visit a dentist and receive medication or surgical treatment, dental claims are not included in the database. And a detailed analysis on the medical cost related to the managements of TN was also not performed. Third, we only evaluated specific medications of interest; however, as these were selected based on expert clinical judgment, we were confident that they were generally representative of the treatments utilized by patients with TN. However, the reasons for medication switches, discontinuation, and treatment outcomes cannot be fully explained with observational studies, and future studies should explore alternative designs to address these questions.

In conclusion, this is the first epidemiological study of $\mathrm{TN}$ in Korea based on the electronic population data from HIRA. The results of this study will not only help to understand the characteristics of TN in Korea, but also serve as an important basis for patients to receive an accurate diagnosis and effective treatment with less cost. Based on our study results, further study, focused on the individual characteristics of TN with large population data, is needed, which would be useful for both patients and physicians.

\section{CONFLICT OF INTEREST}

No potential conflict of interest relevant to this article was reported.

\section{FUNDING}

This study was supported by Wonkwang University in 2019.

\section{ORCID}

Cheol-Hyeong Lee, https://orcid.org/0000-0003-2483-2613

Ho-Yeon Jang, https:/orcid.org/0000-0002-7348-1103

Hyung-Sun Won, https://orcid.org/0000-0001-6084-6698

Ja-Sook Kim, https://orcid.org/0000-0002-2082-6817

Yeon-Dong Kim, https://orcid.org/0000-0003-0404-2657

\section{REFERENCES}

1. Headache Classification Committee of the International Headache Society (IHS) The International Classification of Headache Disorders, 3rd edition. Cephalalgia 2018; 38: 1-211.

2. Love S, Coakham HB. Trigeminal neuralgia: pathology and pathogenesis. Brain 2001; 124(Pt 12): 2347-60.
3. Adams CB. Microvascular compression: an alternative view and hypothesis. J Neurosurg 1989; 70: 1-12.

4. MacDonald BK, Cockerell OC, Sander JW, Shorvon SD. The incidence and lifetime prevalence of neurological disorders in a prospective community-based study in the UK. Brain 2000; 123 (Pt 4): 665-76.

5. Tölle T, Dukes E, Sadosky A. Patient burden of trigeminal neuralgia: results from a cross-sectional survey of health state impairment and treatment patterns in six European countries. Pain Pract 2006; 6: 153-60.

6. Gronseth G, Cruccu G, Alksne J, Argoff C, Brainin M, Burchiel K, et al. Practice parameter: the diagnostic evaluation and treatment of trigeminal neuralgia (an evidence-based review): report of the Quality Standards Subcommittee of the American Academy of Neurology and the European Federation of Neurological Societies. Neurology 2008; 71: 1183-90.

7. Marbach JJ, Lund P. Depression, anhedonia and anxiety in temporomandibular joint and other facial pain syndromes. Pain 1981; 11: 73-84.

8. Han SH, Lee KH, Kim ME, Kim KS. Treatment pattern of patients with neuropathic pain in Korea. J Oral Med Pain 2009; 34: 197-205.

9. Holland M, Noeller J, Buatti J, He W, Shivapour ET, Hitchon PW. The cost-effectiveness of surgery for trigeminal neuralgia in surgically naïve patients: a retrospective study. Clin Neurol Neurosurg 2015; 137: 34-7.

10. El-Tallawy HN, Farghaly WM, Rageh TA, Shehata GA, Abdel Hakeem M N, Badry R, et al. Prevalence of trigeminal neuralgia in Al-Quseir city (Red sea Governorate), Egypt. Clin Neurol Neurosurg 2013; 115: 1792-4.

11. Mueller D, Obermann M, Yoon MS, Poitz F, Hansen N, Slomke MA, et al. Prevalence of trigeminal neuralgia and persistent idiopathic facial pain: a population-based study. Cephalalgia 2011; 31: 1542-8.

12. Lee YS, Kim NH, Sohn HS, Choi KE, Shin HT. Development of drug utilization review guidelines for therapeutic duplication of antipyretics, analgesics, and anti-inflammatory drugs registered in Korea. Korean J Clin Pharm 2010; 20: 21320.

13. De Toledo IP, Conti Réus J, Fernandes M, Porporatti AL, Peres MA, Takaschima A, et al. Prevalence of trigeminal neuralgia: a systematic review. J Am Dent Assoc 2016; 147: 570-6.e2.

14. Katusic S, Beard CM, Bergstralh E, Kurland LT. Incidence and clinical features of trigeminal neuralgia, Rochester, Minnesota, 1945-1984. Ann Neurol 1990; 27: 89-95.

15. Hall GC, Carroll D, Parry D, McQuay HJ. Epidemiology and treatment of neuropathic pain: the UK primary care perspective. Pain 2006; 122: 156-62.

16. Koopman JS, Dieleman JP, Huygen FJ, de Mos M, Martin CG, Sturkenboom MC. Incidence of facial pain in the general population. Pain 2009; 147: 122-7.

17. Bangash TH. Trigeminal neuralgia: frequency of occurrence 
in different nerve branches. Anesth Pain Med 2011; 1: 70-2.

18. Rozen TD. Trigeminal neuralgia and glossopharyngeal neuralgia. Neurol Clin 2004; 22: 185-206.

19. Girard-Tremblay L, Auclair V, Daigle K, Léonard G, Whittingstall K, Goffaux P. Sex differences in the neural representation of pain unpleasantness. J Pain 2014; 15: 867-77.

20. Cui W, Yu X, Zhang H. The serotonin transporter gene polymorphism is associated with the susceptibility and the pain severity in idiopathic trigeminal neuralgia patients. J Headache Pain 2014; 15: 42.

21. Bang S, Kim YS, Lee S, Park U, Kim TK, Choi Y. Prevalence of common causes of neuropathic pain in Korea: population-based observational study. J Int Med Res 2020; 48: 300060519888102.

22. Cruccu G, Gronseth G, Alksne J, Argoff C, Brainin M, Burchiel $\mathrm{K}$, et al. AAN-EFNS guidelines on trigeminal neuralgia management. Eur J Neurol 2008; 15: 1013-28.

23. Maarbjerg S, Di Stefano G, Bendtsen L, Cruccu G. Trigeminal neuralgia - diagnosis and treatment. Cephalalgia 2017; 37:
648-57.

24. Di Stefano G, La Cesa S, Truini A, Cruccu G. Natural history and outcome of 200 outpatients with classical trigeminal neuralgia treated with carbamazepine or oxcarbazepine in a tertiary centre for neuropathic pain. J Headache Pain 2014; 15: 34 .

25. Feller L, Khammissa RAG, Fourie J, Bouckaert M, Lemmer J. Postherpetic neuralgia and trigeminal neuralgia. Pain Res Treat 2017; 2017: 1681765.

26. Moore RA, Chi CC, Wiffen PJ, Derry S, Rice AS. Oral nonsteroidal anti-inflammatory drugs for neuropathic pain. Cochrane Database Syst Rev 2015; 2015: CD010902.

27. Galluzzi KE. Management of neuropathic pain. J Am Osteopath Assoc 2005; 105(9 Suppl 4): S12-9.

28. Dowell D, Haegerich TM, Chou R. CDC guideline for prescribing opioids for chronic pain--United States, 2016. JAMA 2016; 315: 1624-45.

29. Fornasari D. Pharmacotherapy for neuropathic pain: a review. Pain Ther 2017; 6(Suppl 1): 25-33. 